\title{
PERTURBATION APPROACH FOR NUCLEAR MAGNETIC RESONANCE SOLID-STATE QUANTUM COMPUTATION
}

\author{
G. P. BERMAN, D. I. KAMENEV, AND V. I. TSIFRINOVICH
}

Received 22 October 2001 and in revised form 11 July 2002

A dynamics of a nuclear-spin quantum computer with a large number ( $L=1000)$ of qubits is considered using a perturbation approach. Small parameters are introduced and used to compute the error in an implementation of an entanglement between remote qubits, using a sequence of radio-frequency pulses. The error is computed up to the different orders of the perturbation theory and tested using exact numerical solution.

\section{Introduction}

The different solid-state quantum systems are considered now as candidates for quantum computation. They include: nuclear spins $[2,9,14]$, electron spins [3, 8, 13], quantum dots [10], Josephson junctions [1, 11], and others. For the most effective quantum information processing a quantum computer (QC) should operate with a large number of qubits. For numerical simulation of quantum dynamics of this system we must solve a large set of $2^{L}$ linear differential equations for long enough time, or diagonalize many large matrices of the size $2^{L} \times 2^{L}$. Hence, it is important to develop a consistent perturbation theory for quantum computation which allows to predict a probability of correct implementation of the quantum logic operations involving large number of qubits in the real physical systems.

In this paper, we describe the procedure which allows to estimate the errors in implementation of quantum logic operations in the onedimensional solid-state system of nuclear spins, without exact solution of quantum dynamical equations and without direct diagonalization of 


\section{Perturbation approach for NMR QC}

large matrices. We suppose that our computer operates at the temperature $T=0$ and the error is generated only as a result of "internal decoherence" (nonresonant processes). Our approach provides the tools to choose the optimal parameters for operation of the scalable quantum computer with a large number of qubits.

\section{Dynamics of a spin chain}

Application of Ising spin systems for quantum computations was first suggested in [3]. Today, Ising spin systems are used in liquid nuclear magnetic resonance (NMR) quantum computation with small number of qubits [7]. The register (a 1D chain of $L$ identical nuclear spins) is placed in a magnetic field,

$$
\mathbf{B}^{(n)}(z, t)=\left(b_{\perp}^{(n)} \cos \left[v^{(n)} t+\varphi^{(n)}\right],-b_{\perp}^{(n)} \sin \left[v^{(n)} t+\varphi^{(n)}\right], B^{z}(z)\right),
$$

where $t_{n} \leq t \leq t_{n+1}, n=1, \ldots, M, B^{z}(z)$ is a slightly nonuniform magnetic field (with a constant gradient) oriented in the positive $z$-direction, $b_{\perp}^{(n)}$, $v^{(n)}$, and $\varphi^{(n)}$ are, respectively, the amplitude, the frequency, and the initial phase of the circular polarized (in the $x-y$ plane) magnetic field. This magnetic field has the form of rectangular pulses of the length (duration time) $\tau_{n}=t_{n+1}-t_{n}$. The magnetic dipole field on nucleus $k$ in any stationary state is much less than the external field. So, only the $z$ component of the dipole field $B_{d}^{z}(k)$ affects the energy spectrum,

$$
B_{d}^{z}(k)=\sum_{j=0}^{L-1} \frac{3 \cos ^{2} \theta-1}{r_{j k}^{3}} M_{j}^{z},
$$

where $M_{j}^{z}$ is the $z$ component of the $j$ th nuclear magnetic moment, and $r_{j k}$ is the distance between the nuclei $j$ and $k$. In order to suppress the dipole interaction between the spins, we should choose the angle $\theta \approx$ $54.7^{\circ}$ between directions of the spin chain and the permanent magnetic field ( $z$ direction). For this angle $\cos \theta=1 / \sqrt{3}$, and for any stationary state the $z$ component of the dipole field disappears [4].

The Hamiltonian of the spin chain in the magnetic field is

$$
\begin{aligned}
H^{(n)}= & -\sum_{k=0}^{L-1} \omega_{k} I_{k}^{z}-2 J \sum_{k=0}^{L-1} I_{k}^{z} I_{k+1}^{z}-\Theta^{(n)}(t)\left(\frac{\Omega_{n}}{2}\right) \\
& \times \sum_{k=0}^{L-1}\left\{I_{k}^{-} \exp \left[-i\left(v^{(n)} t+\varphi^{(n)}\right)\right]+I_{k}^{+} \exp \left[i\left(v^{(n)} t+\varphi^{(n)}\right)\right]\right\} \\
= & H_{0}+V^{(n)}(t)
\end{aligned}
$$


where the index $k$ labels the spins in the chain, $J$ is the Ising interaction constant, $I_{k}^{z}$ is the operator of $z$ component of $k$ th spin $1 / 2, I_{k}^{ \pm}=I_{k}^{x} \pm i I_{k}^{y}$, $\omega_{k}=\gamma B^{z}\left(z_{k}\right)$ is the Larmor frequency of the $k$ th spin, $\Omega_{n}=\gamma b_{\perp}^{(n)}$ is the precession (Rabi) frequency, $\gamma$ is the gyromagnetic ratio, and we put $\hbar=$ 1 for the Planck constant. The function $\Theta^{(n)}(t)$ equals 1 only during the $n$th pulse and equals zero otherwise. Since the permanent magnetic field has a constant gradient, the frequency difference $\delta \omega=\omega_{k+1}-\omega_{k}$ is the same for all $k$. Below $\delta \omega$ is considered as a parameter of the model.

In order to remove the time-dependence from the Hamiltonian (2.3), we write the wave function $\Psi(t)$ in the time interval of the $n$th pulse, in the laboratory system of coordinates in the form

$$
\begin{aligned}
\Psi(t) & =\exp \left\{i\left[v^{(n)} t+\varphi^{(n)}\right] \sum_{k=0}^{L-1} I_{k}^{z}\right\} \Psi_{\operatorname{rot}}^{(n)}(t) \\
& =\sum_{p} A_{p}(t)|p\rangle \exp \left[-i \chi_{p}^{(n)} t+i \xi_{p}^{(n)}\right]
\end{aligned}
$$

where $\Psi_{\text {rot }}^{(n)}(t)$ is the wave function in a frame rotating with the frequency $\mathcal{v}^{(n)}, x_{p}^{(n)}=-\left[\mathcal{v}^{(n)} / 2\right] \sum_{k=0}^{L-1} \sigma_{k^{\prime}}^{p} \xi_{p}^{(n)}=\left[\varphi^{(n)} / 2\right] \sum_{k=0}^{L-1} \sigma_{k}^{p}, \sigma_{k}^{p}=-1$ if the $k$ th spin of the state $|p\rangle$ is in the position $|1\rangle$, and $\sigma_{k}^{p}=1$ if the $k$ th spin is in the position $|0\rangle$, and $|p\rangle$ is the eigenfunction of the Hamiltonian $H_{0}$.

The dynamics during the $n$th pulse are described by the Schrödinger equation

$$
i \dot{\Psi}=H^{(n)} \Psi
$$

Now, we show that in representation (2.4) the effective Hamiltonian, which describes the dynamics of the system, is independent of time. Consider the matrix elements for the transitions associated with a flip of only one spin, for example, the transition between the states $\left|p_{1}\right\rangle=$ $\left|1_{2} 0_{1} 1_{0}\right\rangle$ and the state $\left|p_{2}\right\rangle=\left|1_{2} 0_{1} 0_{0}\right\rangle$ under the influence of the wave with the frequency $v$ and the initial phase $\xi=\varphi=0$. We have $X_{p_{1}}=v / 2$ and $x_{p_{2}}=-v / 2$, and the matrix element for this transition,

$$
-\left(\frac{\Omega}{2}\right)\left\langle 101\left|e^{i v t / 2} I_{0}^{-} e^{-i v t} e^{i v t / 2}\right| 100\right\rangle=-\frac{\Omega}{2},
$$

is independent of time. In a similar way we can show that matrix elements for other one-spin-flip transitions in representation (2.4) are also time-independent and independent of phase $\varphi^{(n)}$. In brief, in representation (2.4) each spin flip is accompanied by a change of the phase of the wave function by the value $\pm\left[\mathcal{v}^{(n)} t+\varphi^{(n)}\right]$, which compensates the timedependent phase in the perturbation $V^{(n)}(t)$ in the Hamiltonian (2.3). 
The unitary transformation (2.4) is the quantum transformation to the frame rotating with the frequency of the external field $v^{(n)}$ (see, e.g., [5, Chapter 15]).

As follows from (2.3), (2.4), and (2.5) the Schrödinger equation for the coefficients $A_{p}(t)$ has the form

$$
i \dot{A}_{p}(t)=\left[E_{p}-x_{p}^{(n)}\right] A_{p}(t)-\frac{\Omega}{2} \sum_{p^{\prime}} A_{p^{\prime}}(t),
$$

where the sum is taken over the states $\left|p^{\prime}\right\rangle$ related by a single-spin transition with the state $|p\rangle, E_{p}$ is the eigenvalue of the Hamiltonian $H_{0}$, the transition matrix elements are time-independent and equal to each other, as described in the previous paragraph. We can see from (2.7) that the dynamics of the coefficients $A_{p}(t)$ is governed by the effective time-independent Hamiltonian $\mathscr{H}^{(n)}$, and (2.7) can be written in the form $i \dot{A}_{p}(t)=\sum_{p^{\prime}} \mathscr{L}_{p p^{\prime}}^{(n)} A_{p^{\prime}}(t)$, where

$$
\mathscr{H}_{p p^{\prime}}^{(n)}=\left[E_{p}-x_{p}^{(n)}\right] \delta_{p p^{\prime}}-\frac{\Omega}{2} \alpha_{p p^{\prime}} .
$$

Here $\alpha_{p p}=0, \alpha_{p p^{\prime}}=1$ if the states $p$ and $p^{\prime}$ are related to each other by a one-spin-flip transition, and $\alpha_{p p^{\prime}}=0$ for all other states.

The dynamics of the coefficients $A_{p}(t)$ generated by the Hamiltonian $\mathscr{L}^{(n)}$ can be computed using the eigenfunctions $A_{m}^{q(n)}$ and the eigenvalues $e_{q}^{n}$ of this Hamiltonian by

$$
A_{m}\left(t_{n}\right)=\sum_{m_{0}} A_{m_{0}}\left(t_{n-1}\right) \sum_{q} A_{m_{0}}^{q(n)} A_{m}^{q(n)} \exp \left(-i e_{q}^{n} \tau_{n}\right)
$$

Since different pulses of the protocol can have different frequencies and duration times, we should operate in the laboratory frame and make the transformation of the wave function to the rotating frame before each pulse, and the transformation to the laboratory frame after each pulse. If we write the wave function in the laboratory frame in the form

$$
\Psi(t)=\sum_{p} C_{p}(t)|p\rangle \exp \left(-i E_{p} t\right)
$$

then the coefficients $C_{p}(t)$ in (2.10) in the laboratory frame are expressed through the coefficients $A_{p}(t)$ in (2.4) in the rotating frame as

$$
C_{p}(t)=\exp \left[i \mathcal{\xi}_{p}^{(n)} t+i \xi_{p}^{(n)}\right] A_{p}(t)
$$


Here $\varepsilon_{p}^{(n)}=E_{p}-x_{p}^{(n)}$ are the diagonal elements of the Hamiltonian matrix $\mathscr{\ell}_{p p^{\prime}}^{(n)}, t=t_{n-1}$ before the $n$th pulse, and $t=t_{n}=t_{n-1}+\tau_{n}$ after the $n$th pulse. Hereafter we take $\varphi^{(n)}=\xi_{p}^{(n)}=0$ for all $n$.

\section{Two-level approximation}

We explain in this section how selective transitions (resonant transitions), which realize a quantum logic gate, can be implemented in the system described by the Hamiltonian (2.3). To do this, we consider the structure of the effective time-independent Hamiltonian matrix $\mathscr{\ell}_{p p^{\prime}}$. (Here and below we omit the upper index (n).) All nonzero nondiagonal matrix elements are the same and equal to $-\Omega / 2$. At $\Omega \ll \delta \omega$ the absolute values of the diagonal elements in general case are much larger than the absolute values of the off-diagonal elements, and the resonance is coded in the structure of the diagonal elements of the Hamiltonian matrix $\varkappa_{p p^{\prime}}$.

Suppose that we want to flip the $k$ th spin in the chain. To do this, we choose the frequency of the pulse to be equal to the difference $v=E_{p}-E_{m}$ between the energies of the states which related to each other by a flip of the $k$ th spin. The whole Hamiltonian matrix has the form

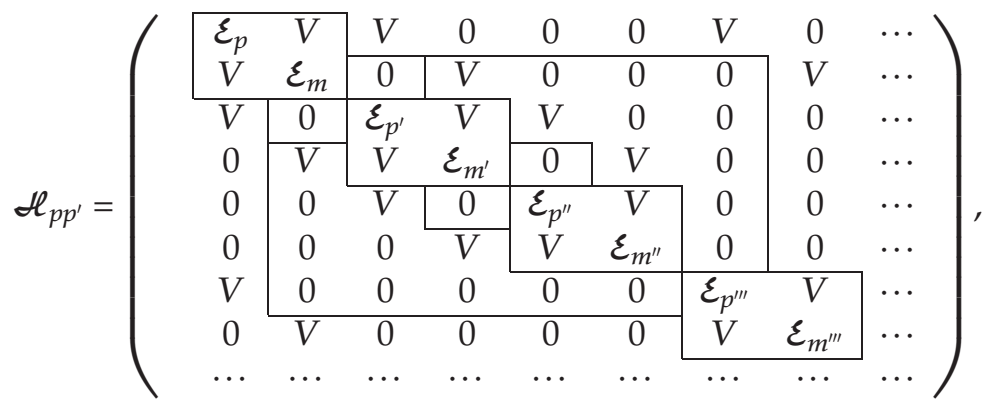

where $\varepsilon_{p}=\varepsilon_{m}+\Delta_{p m},\left|\Delta_{p m}\right| \sim J$ (near-resonance case) or $\left|\Delta_{p m}\right|=0$ (exact resonance case) is the detuning from the resonance, which depends on the positions of the $(k-1)$ th and $(k+1)$ th spins, $V=-\Omega / 2$. All quantum states in this matrix are renumerated in such a way that each two states with the diagonal elements $\boldsymbol{\varepsilon}_{p}$ and $\boldsymbol{\varepsilon}_{m}$, which are related to each other by the resonance or near-resonance transition, are neighbors (i.e., the diagonal elements $\varepsilon_{p}$ and $\varepsilon_{m}$ are close to each other). The different $2 \times 2$ blocks can be organized in an arbitrary order. The states of the different blocks in (3.1) are connected to each other (these relations are indicated by the solid lines in (3.1)) if they differ by a flip of a nonresonant spin, while the other spins are identical. For example, the state $\varepsilon_{p}$ in (3.1) differs from the state $\varepsilon_{m}$ by a flip of a resonant (or near-resonant) spin, while the 
same state $\varepsilon_{p}$ differs from the state $\varepsilon_{p^{\prime \prime \prime}}$ by a flip of a nonresonant spin, and the state $\varepsilon_{p}$ differs from the state $\varepsilon_{p^{\prime \prime}}$ by flips of two nonresonant spins, and so on.

As an illustration we build the matrix (3.1) for the system of three qubits. In this case, we have the matrix of the size $2^{3} \times 2^{3}=8 \times 8$. Suppose that we organize the resonant transition

$$
|p\rangle=\left|0_{2} 0_{1} 0_{0}\right\rangle \longrightarrow|m\rangle=|001\rangle .
$$

Suppose also that

$$
\omega_{0}=\delta \omega, \quad \omega_{1}=2 \delta \omega, \quad \omega_{2}=3 \delta \omega,
$$

and the Ising interaction constant is $J$. Then, the resonant frequency is

$$
v=E_{m}-E_{p}=\frac{1}{2}(\delta \omega-2 \delta \omega-3 \delta \omega)-\frac{1}{2}(-\delta \omega-2 \delta \omega-3 \delta \omega-2 J)=\delta \omega+J
$$

The diagonal elements for these states are

$$
\begin{aligned}
& \varepsilon_{p}=E_{p}-\left(-\frac{3}{2} v\right)=-\frac{3}{2} \delta \omega+\frac{1}{2} J \\
& \varepsilon_{m}=E_{m}-\left(-\frac{1}{2} v\right)=-\frac{3}{2} \delta \omega+\frac{1}{2} J=\varepsilon_{p} .
\end{aligned}
$$

In a similar way we can calculate other diagonal elements. The states in the whole matrix (3.1) correspond to the following quantum states with the corresponding diagonal elements:

$$
\begin{aligned}
|p\rangle=|000\rangle, & \varepsilon_{p}=-\frac{3}{2} \delta \omega+\frac{1}{2} J, & |m\rangle=|001\rangle, & \varepsilon_{m}=-\frac{3}{2} \delta \omega+\frac{1}{2} J, \\
\left|p^{\prime}\right\rangle=|010\rangle, & \varepsilon_{p^{\prime}}=-\frac{1}{2} \delta \omega+\frac{3}{2} J, & \left|m^{\prime}\right\rangle=|011\rangle, & \varepsilon_{m^{\prime}}=-\frac{1}{2} \delta \omega-\frac{1}{2} J, \\
\left|p^{\prime \prime}\right\rangle=|110\rangle, & \varepsilon_{p^{\prime \prime}}=\frac{3}{2} \delta \omega-\frac{1}{2} J, & \left|m^{\prime \prime}\right\rangle=|111\rangle, & \varepsilon_{m^{\prime \prime}}=\frac{3}{2} \delta \omega-\frac{5}{2} J, \\
\left|p^{\prime \prime \prime}\right\rangle=|100\rangle, & \varepsilon_{p^{\prime \prime \prime}}=\frac{1}{2} \delta \omega+\frac{1}{2} J, & \left|m^{\prime \prime \prime}\right\rangle=|101\rangle, & \varepsilon_{m^{\prime \prime \prime}}=\frac{1}{2} \delta \omega+\frac{1}{2} J .
\end{aligned}
$$

In order to find the state $|m\rangle$, which forms a $2 \times 2$ block with a definite state $|p\rangle$, we should flip the resonant spin of the state $|p\rangle$. In other words, positions of $N-1$ (nonresonant) spins of these states are equivalent, while position of the resonant spin is different. 
Under the condition $J \ll \delta \omega=\omega_{k+1}-\omega_{k}$, the energy separation between the diagonal elements of a single $2 \times 2$ block is much less than the energy separation between the diagonal elements of the different blocks $\left(\sim\left|k-k^{\prime}\right| \delta \omega\right)$ which are related to each other by a flip of a nonresonant $k^{\prime}$ th $\left(k^{\prime} \neq k\right)$ spin. Some possible nonresonant transitions are indicated in (3.1) by the lines connecting the different $2 \times 2$ blocks. When the matrix elements, $V$, relating different $2 \times 2$ blocks are small, $|V|=|\Omega / 2| \ll \delta \omega$, in the zero-order approximation, we can neglect the nonresonant interaction between the states of the different blocks, and the Hamiltonian matrix $\mathscr{H}_{p p^{\prime}}$ breaks up into $2^{L} / 2$, approximately independent $2 \times 2$ matrices,

$$
\left(\begin{array}{cc}
\varepsilon_{p} & V \\
V & \varepsilon_{m}
\end{array}\right)
$$

This approximation can be called a two-level approximation, since in this case we have relatively $2^{L} / 2$ independent two-level systems. (Quantitatively, the relative independence of the different $2 \times 2$ blocks follows from (5.1) and (6.3), below. Under the condition $\left|v_{q q^{\prime}}\right| \sim \Omega \ll\left|e_{q}^{(0)}-e_{q^{\prime}}^{(0)}\right| \sim$ $\delta \omega$ the corrections due to interactions between different $2 \times 2$ blocks are small.)

We now derive the solution in the two-level approximation. The dynamics is given by (2.9). Since we deal only with a single $2 \times 2$ block of the matrix $\mathscr{d}_{p p^{\prime}}$ (but not with the whole matrix), the dynamics in this approximation are generated only by the eigenstates of one block. The eigenvalues $e_{q}^{(0)}, e_{Q}^{(0)}$ and the eigenfunctions of the $2 \times 2$ matrix (3.7) are (we put $\Delta_{p m}=\Delta$ )

$$
\begin{array}{ll}
e_{q}^{(0)}=\mathcal{E}_{m}+\frac{\Delta}{2}-\frac{\lambda}{2}, \quad\left(\begin{array}{c}
A_{m}^{q(0)} \\
A_{p}^{q(0)}
\end{array}\right)=\frac{1}{\sqrt{(\lambda-\Delta)^{2}+\Omega^{2}}}\left(\begin{array}{c}
\Omega \\
\lambda-\Delta
\end{array}\right), \\
e_{Q}^{(0)}=\mathcal{E}_{m}+\frac{\Delta}{2}+\frac{\lambda}{2}, \quad\left(\begin{array}{c}
A_{m}^{Q(0)} \\
A_{p}^{Q(0)}
\end{array}\right)=\frac{1}{\sqrt{(\lambda-\Delta)^{2}+\Omega^{2}}}\left(\begin{array}{c}
-(\lambda-\Delta) \\
\Omega
\end{array}\right),
\end{array}
$$

where $\lambda=\sqrt{\Omega^{2}+\Delta^{2}}$. Suppose that before the pulse, the system is in the state $|m\rangle$, that is, the conditions

$$
C_{m}\left(t_{0}\right)=1, \quad C_{p}\left(t_{0}\right)=0
$$




\section{Perturbation approach for NMR QC}

are satisfied. After the transformation, (2.11), to the rotating frame we obtain

$$
A_{m}\left(t_{0}\right)=\exp \left(-i \mathcal{\varepsilon}_{m} t_{0}\right) C_{m}\left(t_{0}\right)=\exp \left(-i \mathcal{\varepsilon}_{m} t_{0}\right), \quad A_{p}\left(t_{0}\right)=0
$$

The dynamics is given by (2.9), which in our case takes the form

$$
\begin{aligned}
A_{m}(t) & =A_{m}\left(t_{0}\right)\left\{\left[A_{m}^{q(0)}\right]^{2} \exp \left[-i e_{q}^{(0)} \tau\right]+\left[A_{m}^{Q(0)}\right]^{2} \exp \left[-i e_{Q}^{(0)} \tau\right]\right\} \\
& =\frac{\exp \left[-i\left[\boldsymbol{\varepsilon}_{m} t-(\Delta / 2) \tau\right]\right]}{\Omega^{2}+(\lambda-\Delta)^{2}}\left\{\Omega^{2} e^{-i \lambda \tau / 2}+(\lambda-\Delta)^{2} e^{i \lambda \tau / 2}\right\},
\end{aligned}
$$

where $t=t_{0}+\tau$. Applying the back transformation

$$
C_{m}(t)=\exp \left(i \mathcal{\varepsilon}_{m} t\right) A_{m}(t),
$$

and taking the real and imaginary parts of the expression in the curl brackets, we obtain

$$
C_{m}\left(t_{0}+\tau\right)=\left[\cos \left(\frac{\lambda \tau}{2}\right)+i\left(\frac{\Delta}{\lambda}\right) \sin \left(\frac{\lambda \tau}{2}\right)\right] \exp \left(-\frac{i \tau \Delta}{2}\right) .
$$

For another amplitude we have,

$$
\begin{aligned}
A_{p}(t) & =A_{m}\left(t_{0}\right)\left\{A_{m}^{q(0)} A_{p}^{q(0)} \exp \left[-i e_{q}^{(0)} \tau\right]+A_{m}^{Q(0)} A_{p}^{Q(0)} \exp \left[-i e_{Q}^{(0)} \tau\right]\right\} \\
& =i \frac{\Omega}{\lambda} \exp \left\{-i\left[\varepsilon_{m} t-\frac{\Delta}{2} \tau\right]\right\} \sin \left(\frac{\lambda \tau}{2}\right)
\end{aligned}
$$

Applying the back transformation

$$
C_{p}(t)=\exp \left[i\left(\varepsilon_{m}+\Delta\right) t\right] A_{p}(t),
$$

we obtain,

$$
C_{p}\left(t_{0}+\tau\right)=i\left(\frac{\Omega}{\lambda}\right) \sin \left(\frac{\lambda \tau}{2}\right) \exp \left(i t_{0} \Delta+\frac{i \tau \Delta}{2}\right) .
$$

When $\Delta=0$ (the resonance case) and $\lambda \tau=\pi$ ( $\pi$-pulse), (3.14) and (3.17) describe the complete transition from the state $|m\rangle$ to the state $|p\rangle$. In the near-resonance case, when $\Delta \neq 0$ the transition probability is (here 
we again put the index $n$ indicating the pulse number),

$$
\varepsilon_{n}=\left(\frac{\Omega_{n}}{\lambda_{n}}\right)^{2} \sin ^{2}\left(\frac{\lambda_{n} \tau_{n}}{2}\right)
$$

We can suppress the near-resonant transitions, and make the probability $\varepsilon_{n}$ equal to zero by choosing the Rabi frequency in the form (see $2 \pi k$ method in [4])

$$
\Omega_{n}^{k}=\frac{\left|\Delta_{n}\right|}{\sqrt{4 k^{2}-1}}
$$

The solutions (3.14) and (3.17) can also be derived without transformation to the rotating frame [4]. However, as will be shown below, our description allows to introduce the small parameters and to build the consistent perturbation theory. Using our approach we will compute the dynamics up to different orders of our perturbation theory, and will test our approximate results by using exact numerical solution for small number of qubits. In Section 7, we will apply the perturbation theory to analyze the quantum dynamics during the implementation of a simple quantum logic gate in the spin chain with large number $(L=1000)$ of qubits.

\section{Protocol for creation of entangled state between remote qubits}

Here we schematically describe the protocol (the sequence of pulses) which allows to create the entangled state for the remote qubits in the system described by the Hamiltonian (2.3). The initial state of the system is supposed to be the ground state $|00 \cdots 00\rangle$. The first pulse in our protocol, described by the unitary operator $U_{1}$, creates the superposition of the states $|00 \cdots 00\rangle$ and $|10 \cdots 00\rangle$ from the ground state,

$$
U_{1}|00 \cdots 00\rangle=\frac{1}{\sqrt{2}}(|00 \cdots 00\rangle+i|10 \cdots 00\rangle) .
$$

Other pulses create from this state the entangled state. This procedure is described by the unitary operator $U^{\prime}$,

$$
U^{\prime} \frac{1}{\sqrt{2}}(|00 \cdots 00\rangle+i|10 \cdots 00\rangle)=\frac{1}{\sqrt{2}}\left(e^{i \varphi_{1}}|00 \cdots 00\rangle+e^{i \varphi_{2}}|10 \cdots 01\rangle\right),
$$

where $\varphi_{1}$ and $\varphi_{2}$ are known phases [4]. 


\section{Perturbation approach for NMR QC}

Now, we describe the procedure for realization of the operator $U=$ $U^{\prime} U_{1}$ by the sequence of pulses. Each pulse is characterized by the corresponding unitary operator, $U_{n}$, where $n=1,2, \ldots, 2 L-2$. (The total number of pulses in our protocol is $M=2 L-2$.) The unitary operator of the whole protocol is a product of the unitary operators of the individual pulses, $U=U_{2 L-2} U_{2 L-4} \cdots U_{2} U_{1}$. The first pulse, described by the operator $U_{1}$, is resonant to the transition between the states $|00 \cdots 00\rangle$ and $|10 \cdots 00\rangle$. If we choose the duration of this pulse as $\tau_{1}=\pi /\left(2 \Omega_{1}\right)($ a $\pi / 2$ pulse), then from (3.14) and (3.17) we obtain (4.1). In order to obtain the second term in the right-hand side of (4.2), we choose a sequence of resonant $\pi$-pulses which transforms the state $|10 \cdots 00\rangle$ to the state $|10 \cdots 01\rangle$ by the following scheme:

$$
\begin{aligned}
|1000 \cdots 0\rangle & \longrightarrow|1100 \cdots 0\rangle \longrightarrow|1110 \cdots 0\rangle \longrightarrow|1010 \cdots 0\rangle \longrightarrow|1011 \cdots 0\rangle \\
& \longrightarrow|1001 \cdots 0\rangle \longrightarrow \cdots \longrightarrow|100 \cdots 11\rangle \longrightarrow|100 \cdots 01\rangle .
\end{aligned}
$$

The frequencies of pulses which realize this protocol are: $v^{(2)}=\omega_{L-2}$, $v^{(3)}=\omega_{L-3}, v^{(4)}=\omega_{L-2}-2 J, v^{(5)}=\omega_{L-4}, \ldots, v^{(2 L-3)}=\omega_{0}-J, v^{(2 L-2)}=\omega_{1}$. If we apply the same protocol to the ground state, then with large probability the system will remain in this state, because all transitions from the ground state are nonresonant.

Since the values of the detuning for the near-resonant transitions from the ground state are the same for all pulses, $\Delta_{n}=\Delta=2 J$ (except for the fourth pulse where $\Delta_{4}=4 \mathrm{~J}$ ), in our calculations we take the values of $\Omega_{n}$ to be the same, $\Omega_{n}=\Omega(n \neq 4)$ and $\Omega_{4}=2 \Omega$. In this case, the probabilities of excitation of the ground state (near-resonant transitions), $\varepsilon_{n}$, are independent of $n: \varepsilon_{n}=\varepsilon$, since $\varepsilon_{n}$ depends only on the ratio $\left|\Delta_{n} / \Omega_{n}\right|$. (Here $\varepsilon$ and $\Omega$ are numerical parameters used in simulations presented below.) We can minimize the probability of the near-resonant transitions choosing $\varepsilon=0$.

\section{Errors in creation of an entangled state for remote qubits}

Our matrix approach allows to estimate the error in the quantum logic gate (4.2) caused by flips of nonresonant spins (nonresonant transitions). Consider a transition between the states $|l\rangle$ and $\left|l^{\prime}\right\rangle$ related by a flip of the nonresonant $k^{\prime}$ th spin. The absolute value of the difference between the $l$ th and $l^{\prime}$ th diagonal elements of the matrix $\mathscr{H}_{p p^{\prime}}^{(n)}$ is of order or greater than $\delta \omega$, because they belong to different $2 \times 2$ blocks. Since the absolute values of the matrix elements which relate different $2 \times 2$ blocks are 
small, $|V| \ll \delta \omega$, we can write

$$
\psi_{q}=\psi_{q}^{(0)}+\sum_{q^{\prime}}^{\prime} \frac{v_{q q^{\prime}}}{e_{q}^{(0)}-e_{q^{\prime}}^{(0)}} \psi_{q^{\prime}}^{(0)}
$$

where the prime in the sum indicates that the term with $q^{\prime}=q$ is omitted, $\psi_{q}$ is the eigenfunction of the Hamiltonian $\mathscr{H}$, the $q$ th eigenstate is related to the $l$ th diagonal element, and the $q$ 'th eigenstate is related to the $l^{\prime}$ th diagonal element, $v_{q q^{\prime}}=2 V\left\langle\psi_{q}^{(0)}\left|I_{k^{\prime}}^{x}\right| \psi_{q^{\prime}}^{(0)}\right\rangle$ is the matrix element for the transition between the states $\psi_{q}^{(0)}$ and $\psi_{q^{\prime}}^{(0)}$, the sum over $q^{\prime}$ takes into consideration all possible nonresonant transitions from the state $|l\rangle$. Because the matrix $\mathscr{t}$ is divided into $2^{N-1}$ relatively independent $2 \times 2$ blocks, the energy $e_{q}^{(0)}\left(e_{q^{\prime}}^{(0)}\right)$ and the wave function $\psi_{q}^{(0)}\left(\psi_{q^{\prime}}^{(0)}\right)$ in (5.1) are, respectively, the eigenvalue and the eigenfunction with the amplitudes given by (3.8) and (3.9) of the effective Hamiltonian, $\mathfrak{t}$, in which all elements are equal to zero except for the elements related to a single $2 \times 2$ block.

The probability of a nonresonant transition from the state $|l\rangle$ to the state $\left|l^{\prime}\right\rangle$ related by a flip of the nonresonant $k^{\prime}$ th spin is

$$
P_{l l^{\prime}}=\left|\left\langle l^{\prime} \mid \psi_{q}\right\rangle\right|^{2}
$$

Only one term in the sum in (5.1) contributes to the probability $P_{l l^{\prime}}$. When the block (3.7) is related to the near-resonant transition $(\Delta / \Omega=2 J / \Omega)$, then from (3.8) and (3.9) the eigenfunctions of this block are

$$
\begin{aligned}
& \psi_{q^{\prime}}^{(0)} \approx\left[1-\frac{\Omega^{2}}{32 J^{2}}\right]\left|l^{\prime}\right\rangle+\frac{\Omega}{4 J}\left|m^{\prime}\right\rangle \approx\left|l^{\prime}\right\rangle, \\
& \psi_{Q^{\prime}}^{(0)} \approx-\frac{\Omega}{4 J}\left|l^{\prime}\right\rangle+\left[1-\frac{\Omega^{2}}{32 J^{2}}\right]\left|m^{\prime}\right\rangle \approx\left|m^{\prime}\right\rangle .
\end{aligned}
$$

On the other hand, if this block is related to the resonant transition $(\Delta=$ $0)$, we have

$$
\psi_{q^{\prime}}^{(0)}=\frac{1}{\sqrt{2}}\left(\left|l^{\prime}\right\rangle+\left|m^{\prime}\right\rangle\right), \quad \psi_{Q^{\prime}}^{(0)}=\frac{1}{\sqrt{2}}\left(\left|l^{\prime}\right\rangle-\left|m^{\prime}\right\rangle\right) .
$$


In both cases $v_{q q^{\prime}} \approx V$, so that

$$
P_{l l^{\prime}} \approx\left(\frac{V}{\varepsilon_{l}-\varepsilon_{l^{\prime}}}\right)^{2} \approx\left(\frac{V}{\left|k-k^{\prime}\right| \delta \omega}\right)^{2}
$$

where we put $e_{q}^{(0)} \approx \varepsilon_{l}, e_{q^{\prime}}^{(0)} \approx \varepsilon_{l^{\prime}} ;\left|k-k^{\prime}\right|$ is the distance from the nonresonant $k^{\prime}$ th spin to the resonant $k$ th spin.

The total probability, $\mu_{N-1}$ (here the subscript of $\mu$ stands for the number of the resonant spin), of generation of all unwanted states by the first $\pi / 2$ pulse (see (4.1)) in the result of the nonresonant transitions is

$$
\mu_{N-1}=\mu \sum_{k^{\prime}=0}^{N-2} \frac{1}{\left|N-1-k^{\prime}\right|^{2}}, \quad \mu=\left(\frac{\Omega}{2 \delta \omega}\right)^{2} .
$$

After the first $\pi / 2$ pulse, the probability of the correct result is $D_{1}=$ $1-\mu_{N-1}$. After the second $\pi$ pulse, the probability of the correct result becomes

$$
p_{2}=\frac{1}{2}\left(1-\mu_{N-1}\right)\left(1-\mu_{N-2}\right)+\frac{1}{2}\left(1-\mu_{N-1}\right)\left(1-\mu_{N-2}-\varepsilon\right) .
$$

The probability of error after applying $2 N-2$ pulses is

$$
\begin{aligned}
P= & 1-p_{2 N-2} \\
= & 1-\frac{1}{2}\left(1-\mu_{N-1}\right)\left(1-\mu_{N-2}-\varepsilon\right)\left(1-4 \mu_{N-2}-\varepsilon\right)\left(1-\mu_{0}-\varepsilon\right) \\
& \times \prod_{i=1}^{N-3}\left(1-\mu_{i}-\varepsilon\right)^{2}-\frac{1}{2}\left(1-\mu_{N-2}\right)\left(1-4 \mu_{N-2}\right) \prod_{i=0}^{N-3}\left(1-\mu_{i}\right)^{2},
\end{aligned}
$$

where the factor 4 at $\mu_{N-2}$ appeared because the Rabi frequency of the fourth pulse which addresses the $(N-2)$ th qubit is twice larger $(2 \Omega)$ than the Rabi frequencies of the other pulses $(\Omega$, see the end of Section 4$)$, the square at $\left(1-\mu_{i}\right)^{2}$ and $\left(1-\mu_{i}-\varepsilon_{i}\right)^{2}$ appears because we address the $i$ th qubit twice (see a sequence of the resonant $\pi$-pulses in Section 4 ), and the last two terms in the right-hand side of (5.8) are related to the last two terms in the right-hand side of (4.2). In the approximation described in this section we took into consideration all one-spin-flip transitions because we neglected the terms of the order of $\Omega /(4 J)$ and smaller in (5.3). 


\section{Improved perturbation theory}

In the analysis presented before, we used the approximate solutions (5.3) and (5.4) for the wave functions (3.8) and (3.9). In a more exact approach, which also does not requires diagonalization of the large matrices, we use the explicit forms (3.8) and (3.9) to express the wave functions $\psi_{q^{\prime}}^{(0)}$ and $\psi_{Q^{\prime}}^{(0)}$ of the $2 \times 2$ blocks, in (5.1),

$$
\psi_{q^{\prime}}^{(0)}=A_{m^{\prime}}^{q^{\prime}(0)}\left|m^{\prime}\right\rangle+A_{l^{\prime}}^{q^{\prime}(0)}\left|l^{\prime}\right\rangle, \quad \psi_{Q^{\prime}}^{(0)}=A_{m^{\prime}}^{Q^{\prime}(0)}\left|m^{\prime}\right\rangle+A_{l^{\prime}}^{Q^{\prime}(0)}\left|l^{\prime}\right\rangle .
$$

Then, we put the functions $\psi_{q^{\prime}}^{(0)}$ and $\psi_{Q^{\prime}}^{(0)}$ into (5.1), and obtain the expression for the wave function in the form

$$
\psi_{q}=\sum_{m} A_{m}^{q}|m\rangle,
$$

where the sum in the right-hand side includes $2 L$ terms. Using the functions $A_{m}^{q}$, we solve the dynamical equations (2.9) with the energies $e_{q}$ computed up to the second order of our perturbation theory by

$$
e_{q}=e_{q}^{(0)}+\sum_{q^{\prime}}^{\prime} \frac{\left|v_{q q^{\prime}}\right|^{2}}{e_{q}^{(0)}-e_{q^{\prime}}^{(0)}},
$$

where the prime in the sum means that the term with $q=q^{\prime}$ is omitted, $e_{q}^{(0)}$ is defined by (3.8) or (3.9), and the matrix elements $v_{q q^{\prime}}$ are defined after (5.1).

We call the described approach in this section the improved perturbation theory to indicate the difference from the approach considered in Section 5. In this approximation each eigenfunction $\psi_{q}$ of the Hamiltonian $\mathscr{t}$ is expanded over $2 L$ (see (6.2)) basis functions $|m\rangle$, with all other coefficients $A_{m^{\prime}}^{q}$ being equal to zero. Here we use all possible transitions between different $2 \times 2$ blocks in (3.1) which include the two-spin-flip transitions: a flip of the spin with a near-resonant frequency and a flip of a nonresonant spin. The number of the nonzero coefficients in this approximation is $2^{L} \times 2 L$. It still can be large for large $L$ and can require large computer memory for simulation. As will be shown below, at the condition $\Omega \ll J \ll \delta \omega$ this approach (the improved perturbation theory) provides the results which practically coincide with the exact solution. In this approximation we neglect, for example, the two-step transitions like the transition $\varepsilon_{p} \rightarrow \varepsilon_{p^{\prime \prime}}$ in (3.1) which occurs with the probability $\sim \mu^{2}$ and which is associated with the flips of two nonresonant spins. 


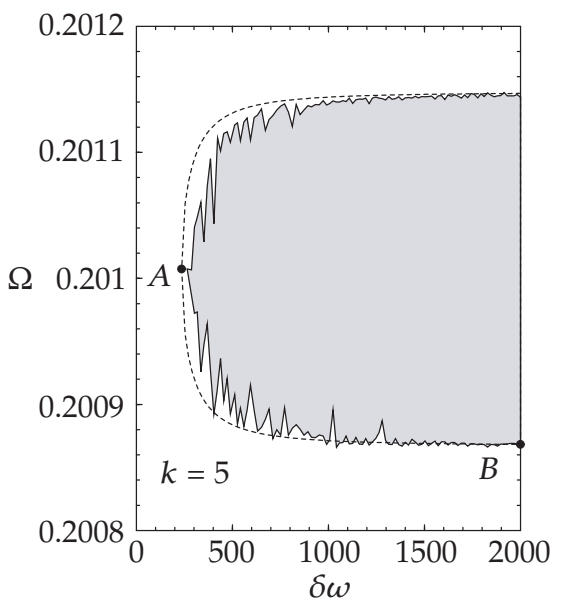

(a)

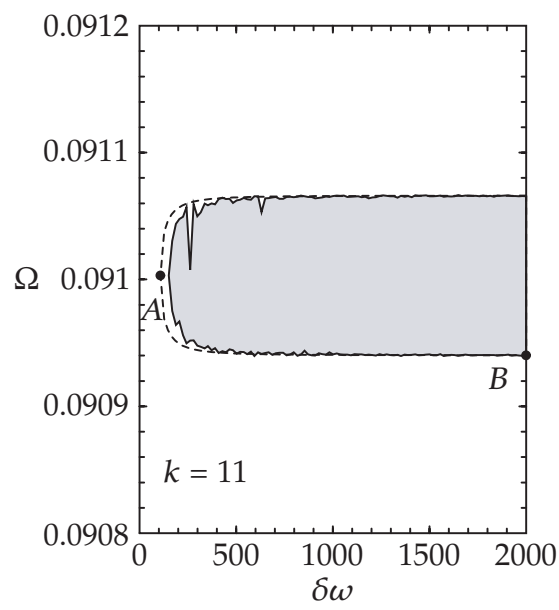

(b)

FIGURE 7.1. The probability of generation of unwanted states $P$ at different values of $\delta \omega$ and $\Omega$. In the gray regions $P<P_{0}, P_{0}=10^{-5}$. The region delimited by the dashed line is obtained using (5.8). The gray region delimited by the solid line is obtained using the improved perturbation theory described in Section 6. The position of the point $A$ in $\Omega$ satisfies the $2 \pi k$ condition (3.19), $\Omega=\Omega^{k}$, where the values of $k$ are indicated in the figures. $L=10$.

\section{Numerical results}

All frequencies in this section are measured in units of $J$. Suppose that we are able to correct the errors with the probability less than $P_{0}=10^{-5}$, using some additional error correction codes. Our perturbation theory allows to calculate the region of parameters for which the probability of error $P$ in realization of the logic gate (4.2) is less than $P_{0}$. In Figures $7.1 \mathrm{a}$ and $7.1 \mathrm{~b}$ we plot the diagrams obtained by solution of $(5.8)$ and using the improved perturbation theory. In the gray areas the probability of generation of unwanted states is less than $P_{0}$. We can see that two approaches provide similar results. They become practically identical at large values of $\delta \omega$.

In almost all quantum algorithms the phase of the wave function is important. We numerically compared the phase of the wave function on the boundaries of the gray regions in Figures 7.1a and 7.1b with the phase in the centers of these regions, where $\Omega$ satisfies $2 \pi k$ condition, 


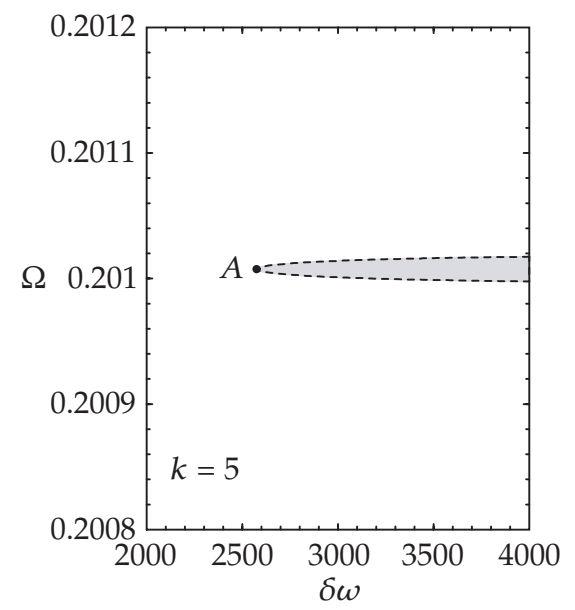

(a)

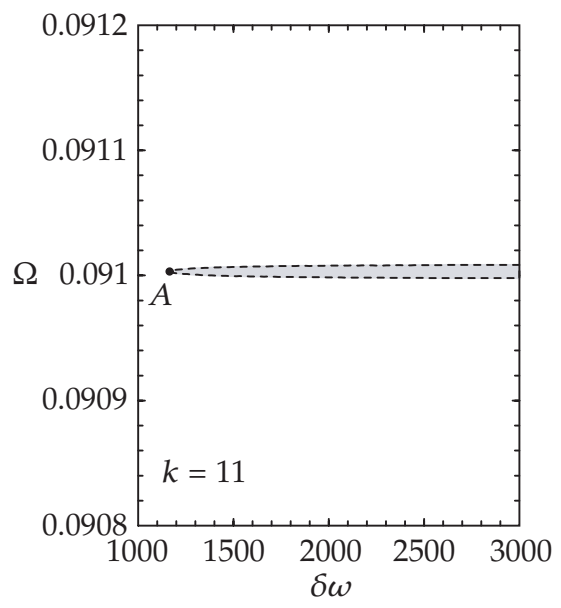

(b)

Figure 7.2. The same as in Figures 7.1a and 7.1b but for $L=1000$.

The error was calculated using (5.8).

and the expression for the phase can be obtained analytically [4]. The deviation in phase is only $\sim 0.15 \%$. This is much less than the corresponding change in the probability, $P$, of error, and we do not consider below the error in the phase of the wave function.

We now analyze the probability of errors as a function of $\delta \omega$. When the value of $\delta \omega$ is large enough, the probability of error (and the widths of the gray areas in $\Omega$ ) becomes practically independent of $\delta \omega$. This is because at $\delta \omega \gg 1$ and at $\varepsilon \gg \mu$ the error is mostly defined by $\varepsilon$, which is independent of $\delta \omega$. As a consequence, we can, for example, estimate the widths of the gray areas at $\delta \omega \gg 1$ taking into account only the nearresonant transitions. To do this, we put in (5.8) the value $\mu_{n}=0$ for all $n$ and obtain

$$
P_{B}=\frac{1}{2}\left[1-\prod_{n=1}^{2 L-3}(1-\varepsilon)\right]=\frac{1}{2}\left[1-(1-\varepsilon)^{2 L-3}\right] \approx \frac{2 L-3}{2} \varepsilon .
$$

The positions of the boundaries in $\Omega$ in Figures 7.1a and 7.1b can be obtained from the equation $P_{B}=P_{0}$, where $P_{B}$ is given by (7.1) and $\varepsilon$ is the function of $\Omega$ (see (3.18)).

We can see from (5.8) that the number of qubits $L$ in our approach is a scalable parameter that can be increased without principal change in the calculation procedure. We used (5.8) to create the error diagrams as in Figures 7.1a and 7.1b but for as much as 1000 qubits in Figures 


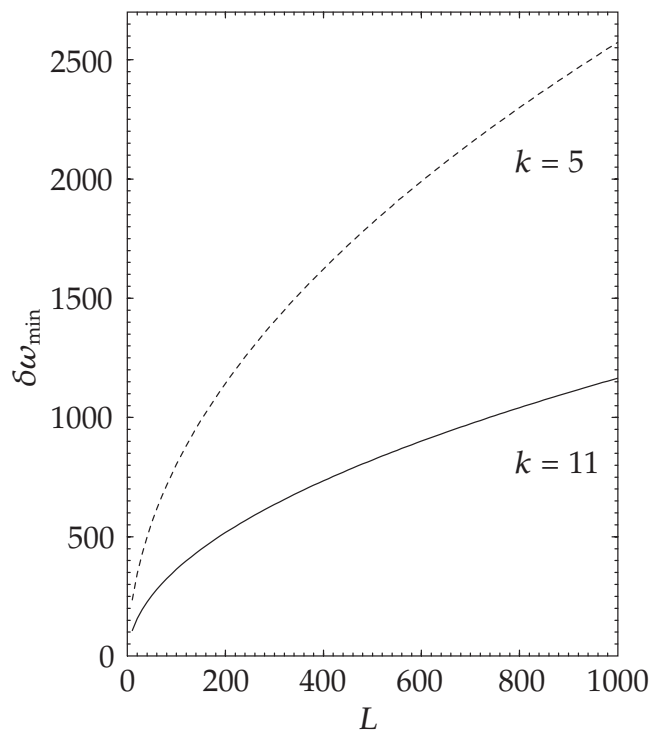

FIgURE 7.3. The minimum value of $\delta \omega, \delta \omega_{\min }=\delta \omega_{A}$ (see Figures 7.1a and 7.1b for $L=10$ and Figures $7.2 \mathrm{a}$ and $7.2 \mathrm{~b}$ for $L=1000$ ), required to make the error in the logic gate (4.2) below the threshold $P_{0}=10^{-5}$, as a function of number of qubits. The value of $\Omega$ in the point $A, \Omega_{A}$, satisfies the conditions of the $2 \pi k$-method, $\Omega_{A}=\Omega_{k}$. The values of $k$ are indicated in the figure.

7.2a and 7.2b. The Hilbert space for this problem contains $2^{1000}$ states, and the exact solution is impossible. In our approach we overcome this problem by taking into consideration only the states with the relatively large probabilities.

In Figures 7.1a, 7.1b, 7.2a, and 7.2b the value of $\Omega$ at the point $A$, $\Omega_{A}$, satisfies the conditions of the $2 \pi k$-method, and near-resonant transitions are completely suppressed $(\varepsilon=0)$. From Figures 7.1a, 7.1b, 7.2a, and $7.2 \mathrm{~b}$, we can see that even in the case when the condition of $2 \pi k$ method is satisfied the error can be large due to the nonresonant transitions which cannot be completely eliminated. The value of $\Omega$ cannot be decreased considerably because decreasing of $\Omega$ makes the quantum computer very slow so that the quantum state can be destroyed by decoherence due to possible influence of environment. Hence, we can make the value of $\mu$ small by increasing $\delta \omega$, or the gradient of the permanent magnetic field. Thus, at $\delta \omega<\delta \omega_{A}$, where $\delta \omega_{A}$ is the coordinate of the point $A$ in $\delta \omega$ in Figures 7.1a, 7.1b, 7.2a, and 7.2b, the error is greater than $P_{0}$. In Figure 7.3 we plot the minimum value of $\delta \omega=\delta \omega_{A}$ as a function of the number of qubits $L$ computed by using (5.8). We can see that $\delta \omega_{A}$ becomes large for large $N$. Thus, for example, 


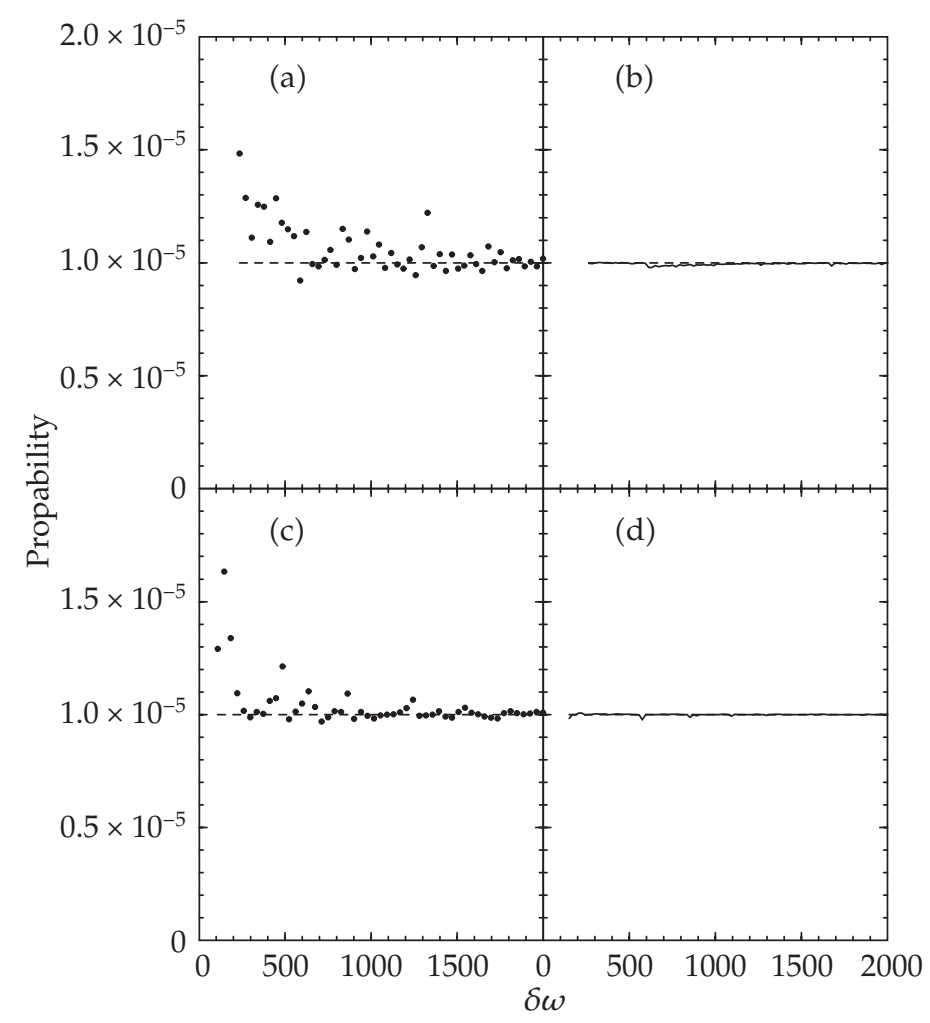

FIGURE 7.4. The exact solution for the probability of generation of unwanted states $P$ computed using the parameters which correspond to (a) dashed curve $A B$ in Figure 7.1a (obtained using $(5.8))$, (b) lower boundary of the hatched region in Figure 7.1a obtained using the improved perturbation theory, (c) dashed curve $A B$ in Figure 7.1b (obtained using (5.8)), (d) lower boundary of the hatched region in Figure 7.1b obtained using the improved perturbation theory. The dashed lines indicate the solutions obtained using (corresponding) perturbation theory for the same parameters.

for protons with $J /(2 \pi) \sim 10 \mathrm{~Hz}$ (for estimations in this paragraph we use the dimensional units) with the distance between the neighboring spins $a=2 \mathrm{~nm}$, the value $\delta \omega / J=1000$ gives the gradient of the magnetic field $\delta \omega /(\gamma a \cos \theta) \sim 2 \times 10^{5} \mathrm{~T} / \mathrm{m}[6,12]$. From Figure 7.3, we can see that this is the minimum value of the gradient of the magnetic field for $L_{\max } \approx 155$ when $\Omega=\Omega^{5}$ and $L_{\max } \approx 740$ when $\Omega=\Omega^{11}$ required to make the error less than $P_{0}=10^{5}$. At $L>L_{\max }$, at a given gradient of the magnetic field, and at $\Omega \approx \Omega^{k}, k=5$ or 11 , the error will be always larger than $P_{0}$. 


\section{Perturbation approach for NMR QC}

In Figures 7.4, we test our perturbation theory by using the exact numerical solution obtained by diagonalization of $2^{L} \times 2^{L}$ matrices and using (2.9). We can see that there is good correspondence with the exact numerical solution for the results obtained using (5.8), and practically exact correspondence for the solution obtained using the improved perturbation theory. The similar correspondence can be demonstrated for other parameters $(\delta \omega, \Omega)$.

\section{Conclusion}

We developed a perturbation theory which allows to estimate the errors in the implementation of the quantum logic gates by the radio-frequency pulses in the solid-state system with large number (1000 and more) of qubits. Our perturbation approach correctly describes the behavior of the quantum system in the large Hilbert space (the Hilbert space with a large number of states) and predicts the final quantum state of the system after action of the sequence of pulses with different frequencies. This is possible because in the system there exist small parameters which characterize the probabilities of the near-resonant transitions, $\varepsilon$, and probabilities of the nonresonant transitions, $\mu$, which are small, $\varepsilon \ll 1$ and $\mu \ll 1$, when the conditions $\Omega \ll J \ll \delta \omega$ are satisfied. Our approach allows to control the quantum logic operations in the system with large number of qubits and to minimize the error caused by the internal decoherence (nonresonant processes).

\section{Acknowledgments}

This work was supported by the Department of Energy (DOE) under contract W-7405-ENG-36, by the National Security Agency (NSA), and by the Advanced Research and Development Activity (ARDA).

\section{References}

[1] D. V. Averin, Adiabatic quantum computation with Cooper pairs, Solid State Commun. 105 (1998), 659-664.

[2] G. P. Berman, G. D. Doolen, P. C. Hammel, and V. I. Tsifrinovich, Solid-state nuclear-spin quantum computer based on magnetic resonance force microscopy, Phys. Rev. B 61 (2000), 14694-14699.

[3] G. P. Berman, G. D. Doolen, D. D. Holm, and V. I. Tsifrinovich, Quantum computer on a class of one-dimensional Ising systems, Phys. Lett. A 193 (1994), 444-450.

[4] G. P. Berman, G. D. Doolen, G. V. López, and V. I. Tsifrinovich, Simulations of quantum-logic operations in a quantum computer with a large number of qubits, Phys. Rev. A (3) 61 (2000), 062305. 
[5] G. P. Berman, G. D. Doolen, R. Mainieri, and V. I. Tsifrinovich, Introduction to Quantum Computers, World Scientific, Singapore, 1998.

[6] K. J. Bruland, W. M. Dougherty, J. L. Garbini, J. A. Sidles, and S. H. Chao, Force-detected magnetic resonance in a field gradient of 250000 Tesla per meter, Appl. Phys. Lett. 73 (1998), 3159-3161.

[7] I. L. Chuang, N. Gershenfeld, and M. Kubinec, Experimental implementation of fast quantum searching, Phys. Rev. Lett. 80 (1998), 3408-3411.

[8] A. Imamoglu, D. D. Awschalom, G. Burkard, D. P. DiVincenzo, D. Loss, M. Sherwin, and A. Small, Quantum information processing using quantum dot spins and cavity-QED, Phys. Rev. Lett. 83 (1999), 4204-4207.

[9] B. E. Kane, A silicon-based nuclear spin quantum computer, Nature 393 (1998), 133-137.

[10] M. Sherwin, A. Imamoglu, and T. Montroy, Quantum computation with quantum dots and terahertz cavity quantum electrodynamics, preprint, 1999, http:/ /xxx.lanl.gov/abs/quant-ph/9903065.

[11] A. Shnirman, G. Schoen, and Z. Hermon, Quantum manipulations of small Josephson junctions, Phys. Rev. Lett. 79 (1997), 2371-2374.

[12] D. Suter and K. Lim, Scalable architecture for spin-based quantum computers with a single type of gate, Phys. Rev. A 65 (2002), 052309.

[13] R. Vrijen, E. Yablonovitch, K. Wang, H. W. Jiang, A. Balandin, V. Roychowdhury, T. Mor, and D. DiVincenzo, Electron-spin-resonance transistors for quantum computing in silicon-germanium heterostructures, Phys. Rev. A 62 (2000), 012306.

[14] F. Yamaguchi and Y. Yamamoto, Crystal lattice quantum computer, Microelectronic Engineering 47 (1999), 273-275.

G. P. Berman: Theoretical Division and CNLS, Los Alamos National Laboratory, Los Alamos, NM 87545, USA

E-mail address: gpb@t13.lanl.gov

D. I. Kamenev: Theoretical Division and CNLS, Los Alamos National Laboratory, Los Alamos, NM 87545, USA

E-mail address: kamenev@cnls.lanl.gov

V. I. Tsifrinovich: Department of Introductory Design and Science, Polytechnic University, Six Metrotech Center, Brooklyn, NY 11201, USA

E-mail address: vtsifrin@duke.poly.edu 


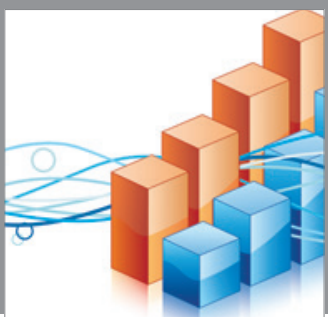

Advances in

Operations Research

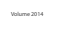

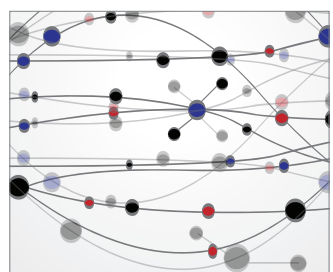

\section{The Scientific} World Journal
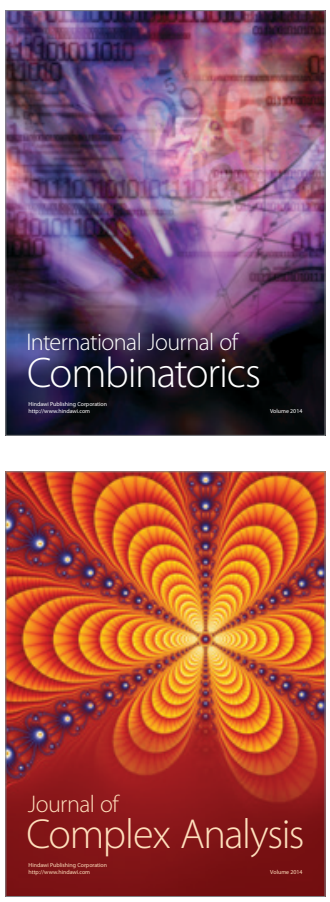

International Journal of

Mathematics and

Mathematical

Sciences
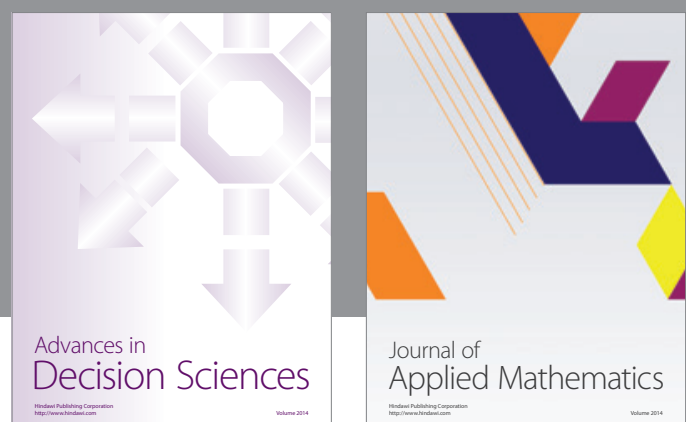

Journal of

Applied Mathematics
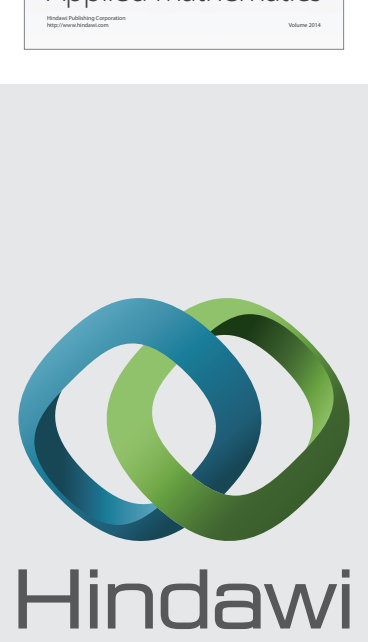

Submit your manuscripts at http://www.hindawi.com
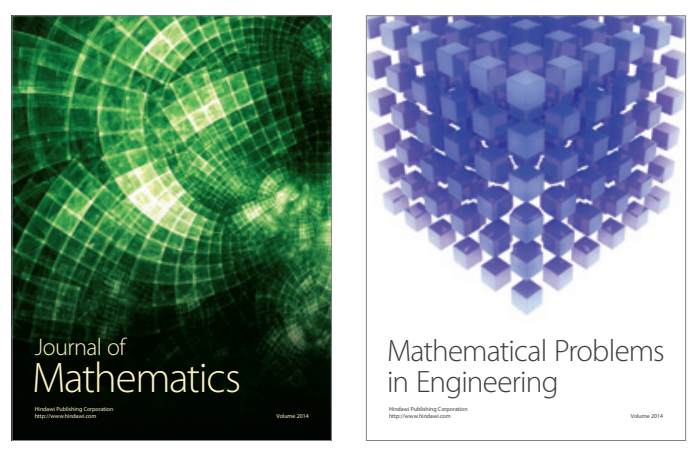

Mathematical Problems in Engineering
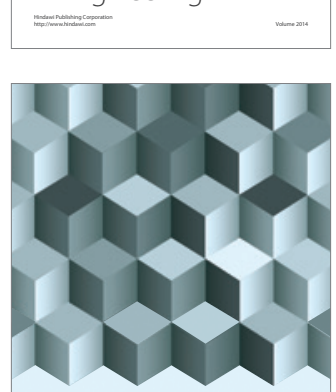

Journal of

Function Spaces
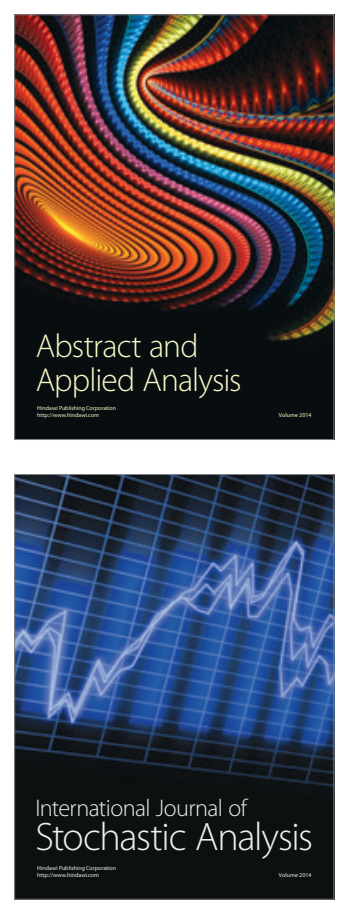

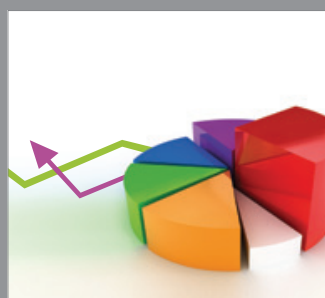

ournal of

Probability and Statistics

Promensencen
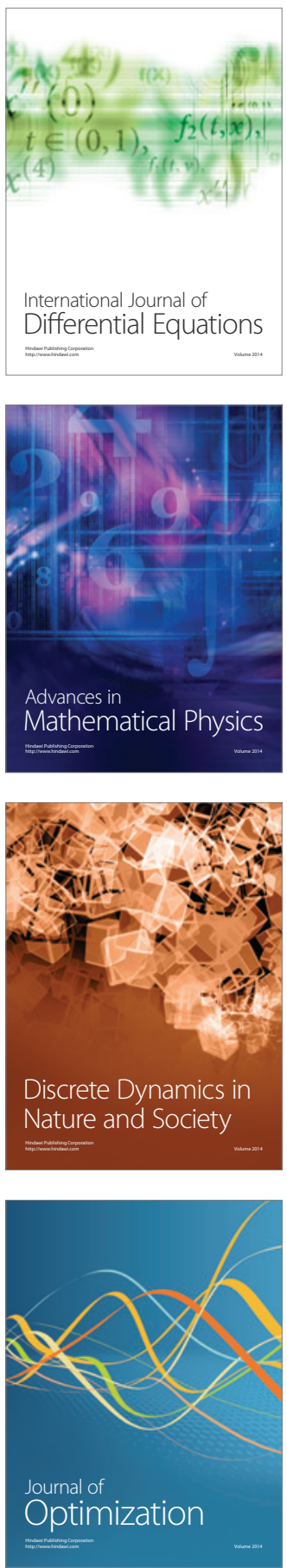